\title{
Comparative Effectiveness Research (CER) in Nigeria: the Milestones and the Millstones.
}

\author{
${ }^{1}$ Dr Enembe ${ }^{2}$ O.Okokon, ${ }^{3}$ Dr Daprim S. Ogaji \\ ${ }^{I}$ Department of Environmental Science, University of Eastern Finland, Kuopio Campus, Kuopio, Finland. \\ ${ }^{2}$ Department of Community Medicine, University of Calabar Teaching Hospital, Calabar. Nigeria. \\ ${ }^{3}$ Department of Community Medicine, Niger Delta University, Wilberforce Island, Bayelsa State. Nigeria.
}

\begin{abstract}
Background: The relevance of empirical evidence in healthcare decision making is well acknowledged. Within any setting constrained by a lean resource base, stronger premium should be placed on evidence which informs policy; as ever so often in such climes, multiplicity of healthcare needs outstrip available resources. Thus, the outstanding challenge is how to best serve the most compelling needs of the teeming populace by a fair balance of evidence and resource outlay within the complex imperatives of the overarching socio-cultural context. Familiar poor development indices underscore most developing countries; peculiarities also exist with respect to national priorities, resource base, available technologies and infrastructure, philosophies and cultures, and penchant for adaptation. Nigeria is one such complex society, trying to address healthcare needs of her citizens while concurrently addressing competing needs from other sectors by a very delicate balancing act. This study aims to provide an overview of the state and turnover of Comparative Effectiveness Research (CER) in Nigeria between the years 1975 and 2009, and the extent to which key health problems are addressed within study imperatives.

Methods: Studies were selected by running searches in PubMed and Cochrane databases. Search terms such as Compar*, effectiv*, research, study, and Nigeria were used. The Cochrane Library was searched using a listing of Nigerian authors provided by the South African Cochrane Centre. Studies were selected both by study design (experimental studies - Randomized Control Trials, Controlled Clinical Trial, analytic studies or full systematic reviews on these designs) and by study setting which in this instance was Nigeria. Data extraction was done using a checklist designed to capture priority areas apparent within the studies reviewed and as enunciated by the National Health Policy.

Results: The overall volume of intervention studies was low. A greater proportion of studies, 48.5\%, investigated treatment options for infectious and parasitic diseases; $24.2 \%$ investigated reproductive health interventions. Approximately $62.6 \%$ of interventions aimed to achieve cure, and $29.3 \%$ aimed for prevention. Study endpoints were efficacy (95.9\%), adverse effects/tolerability (60.6\%), treatment failure (28.6\%) and cost (6.1\%). Study patterns were similar in different geopolitical zones of the country. No stable funding structure could be identified for these studies.
\end{abstract}

Conclusion: CER does reflect the pattern of need and the dominant type of healthcare in Nigeria. Interventions oriented to achieve cure dominate the CER landscape in Nigeria.

Keywords: comparative effectiveness research, healthcare, Nigeria

\section{Background}

The importanceof evidence driven decision making in any setting with limited resources amidst multiplicity of healthcare needs cannot be overstated ${ }^{(1-3)}$. The health needs of the majority will be best served where there is a fair balance of evidence, resource outlay, context and political will. With a broad array of interventions aimed toward promoting, protecting, preserving and restoring of health, there arises the need to streamline research findings with an objective to distill the best available evidence for effective healthcare outcomes. Quite appropriately, this discourse considers 'effectiveness' which is somewhat distinct from the closely related measure of potency of an intervention type, namely 'efficacy'. While efficacy addresses interventional outcome in an ideal setting, effectiveness takes cognizance of constraints and challenges inherent in normative settingswithin which an intervention is applied ${ }^{(4-5)}$. The same constraints may well determine the thrust of native research in these settings.

CER has been succinctly described as research that generates evidence that compares treatment ${ }^{(6)}$. The Institute of Medicine, Washington DC, offers a more robust definition which describes CER as "The generation and synthesis of evidence that compares the benefits and harms of alternative methods to prevent, diagnose, treat, and monitor a clinical condition, or to improve the delivery of care" ${ }^{(7)}$ In other words, it is research that compares one intervention to others aimed at a similar endpoint after these interventions have been approved for market distribution by relevant regulatory authorities. Implied above is the idea that stronger evidence 
Comparative Effectiveness Research (CER) In Nigeria: The Milestones And The Millstones.

supporting the effectiveness of one course of health action over another would form the basis for unbiased decisions ${ }^{(8)}$. Comparative effectiveness research would be a handydecision making and policy tool for addressing the health imperatives of a developing country ${ }^{(1,9-10)}$. Considerations of sustainability of interventions evidenced as ideal for achieving desirable health outcomes should be carried along as worthy objectives of CER studies as well as methods for transmitting research findings to policy frontlines ${ }^{(2,11)}$. Though familiar development indices are shared among low income and lower-middle income countries, peculiarities exist with respect to national priorities, resource base, available technologies and infrastructure, philosophies and cultures, and penchant for adaptation in a world fraught with an expandingpalette of diseases. Using the Nigerian situation as a gauge within the West African sub-region, this study aims to provide a perspective on the state of CER in a developing country setting. The point must be made that different studies on this same topic may highlight different aspects of the situation. Our approach will be to examine the priority CER areas; the fundamental question and issues addressed by such research; and the extent to which such research approximates priorities outlined by policy makers.

Recognition of the pivotal role of research in health decisions is evinced in the $3^{\text {rd }}$ chapter of the 2004 Nigerian National Revised Health Policy, where the $9^{\text {th }}$ heading reads "National Health Planning." One of the bullet points under this heading states that, "The functions inherent in health planning shall be broken into: (i) Research, that is, analytical and descriptive processes resulting in strategic policy choices and long-term objectives, will be a continuous process that cannot appropriately be fitted into an annual cycle, though an annual summary of long-term aims and objectives shall be produced as a background to programming decisions .$^{\prime \prime}{ }^{(12)}$ The $5^{\text {th }}$ chapter of the policy document has the broad heading, "National Health Interventions". Subheadings within this chapter include National Policy on HIV/AIDS; on Roll Back Malaria; on Immunization; on Control of Onchocerciasis; on Control of Tuberculosis and Leprosy; on Blood Transfusion; on Elimination of Female Genital Mutilation; on Reproductive Health; on Adolescent Health; on Food and nutrition; on Child Health; on Drugs; and on Food Hygiene and Safety Practices ${ }^{(12)}$.

\section{Box 1. Nigerian Healthcare Policy Imperatives Potentially Driving Research ${ }^{(12)}$}

Extracts from the Nigerian 2004 Revised National Health Policy as documented under the following headings:

\section{"Health Status}

- Preventable diseases account for most of Nigeria's disease burden and poverty is a major cause of these problems.

- Our maternal mortality rate (about one mother's death in every one hundred deliveries) is one of the highest in the world.

- Some other health indicators, such as under-5 mortality rate, are higher than the average for subSaharan Africa ....

Health Policy, Legislation, and Health Sector Reform Agenda

- There is limited capacity for policy/plan/programme formulation, implementation, monitoring and evaluation at all levels ...

Health Service Delivery and Quality of Care

- Disease programmes, such as HIV/AIDS, TB, malaria and other programmes, such as reproductive health, are currently being implemented within a weak system and have had little impact ...

\section{Health Finance}

- Public expenditure on health is less than $\$ 8$ per capita, compared to the $\$ 34$ recommended internationally. Private expenditures are estimated to be over $70 \%$ of total health expenditure with most of it coming as out-of-pocket expenses in spite of the endemic nature of poverty.

- There is no broad-based health financing strategy."

\section{Methodology}

This desk review of journal articles on CER studies conducted in Nigeria was done in the month of August, 2010. The studies considered were retrieved from PubMed and Cochrane database searches. The PubMed database was searched using the syntax:

(((compar*) AND effectiv*) AND research OR study) OR clinical trial [MeSH Terms]) AND Nigeria [Affiliation] 
Comparative Effectiveness Research (CER) In Nigeria: The Milestones And The Millstones.

The search builder was used in an attempt to streamline search results.Filters such as human studies, English language (English being the national language in Nigeria), randomized control trials, control clinical trials,phase III trials, systematic reviews, and meta-analysis were used. The Cochrane database search was conducted using a full administrative listing of Nigerian authors. Completed reviews by these authors were scrutinized and appropriate data obtained.

\section{Selection criteria for studies were:}

1. Study must have been carried out by a Nigerian author, in a Nigerian setting, with affiliations to a Nigerian institution; or by a foreign author conducting studies pertinent to a setting within Nigeria.

2. Study design: completed systematic reviews, RCT, CCT, analytical study comparing interventions. We draw attention to the fact that even though a distinction has been made between evidence based medicine and CER; for the purpose of this study, we are considering both types of research studies together as our objective is to elucidate patterns rather than specific findings.

Data was extracted using aquestionnaire/checklist developed by the reviewers. The key areas of interest were:

- the broad classification of disease/conditions informing interventions

- specific conditions being intervened upon

- the geopolitical zone withinNigeria where the principalinvestigator is based or where the research study held

- intervention type e.g. diagnostic, preventive etc.

- intervention end-points.

The diseases/conditions investigated in the studies were broadly classified based on the ICD $10^{\text {th }}$ revision. This was modified to accommodate conditions which were not diseases per se. Some conditions researched were interventions aimed at health promotion. Individual diseases were also noted during the data extraction. Limitations: 1.Being that only two electronic databases were searched, out of many possible sources of electronic and grey literatures, a possible selection bias may not be discountenanced in the emergent patterns.

2. The focus was on highlighting patterns; thus only summary estimates were used. Due to the low overall volume of CER, and the disparity between different Nigerian geopolitical zones, attaching inferential value to obtained data may only yield statistical artifacts and nothing more.

\section{Results}

The PubMed search yielded 6214hits. Repeated studies, expert reviews, and largely multinational studies were weeded out leaving 376 studies of which 89 met the inclusion criteria. Use of the search builder yielded no further studies. Additional search of the Cochrane library (using the list of Nigerian authors) yielded 10 more studies, giving a total of 99 studies. Studies includedSystematic Reviews -18(18.2\%); RCTs 54(54.5\%); CCTs -21(21.2\%); Analytical Studies -6 (6.1\%).

Diseases and Conditions Researched:

Broad categorization of researched diseases showed that infections/parasitic diseases were the most researched $(48.5 \%)$, followed by reproductive health concerns $(24.2 \%)$, and thirdly cardiovascular (CV) conditions $(8 \%)$ (Table 1).

Specific diseases/conditions investigated undercardiovascular, reproductive, and infections/infestations categories are shown in Figures 1, 2 and 3. Other disease conditions wereAllergic Rhinitis (1.0\%), Anaemia(1.0\%), Appendicitis (1.0\%), Asthma (1.0\%), CA Cervix (1.0\%), Dental Caries (1.0\%), Depression (1.0\%), Diarrhoea (2.0\%), Endodontic Flare-ups (1.0\%), Eye Care (1.0\%), Eye Condition (1.0\%), Joint Pain (1.0\%), Kwashiorkor (1.0\%), Osteoarthritis (1.0\%), Periodontitis (1.0\%), Pruritus (1.0\%), Pterygium (2.0\%), Snake Bite (3.1\%).

Of the 48 studies examining infectious/parasitic conditions, there were 23 malaria studies which made up $47.9 \%$ of total, while HIV/AIDS related studies made up 6.3\% (Figure 1). Twenty-four research studies were interested in female reproductive health; 10 studies on contraception made up 41.7\%; studies aimed at improving conduct of labour were 7(29.2\%), and studies on interventions to prevent post-partum haemorrhage contributed $12.5 \%$ (Figure 2).

Conditions Researched by Geopolitical Zones

Designating summary estimates of study interests by zones showed that the highest volume (62 studies in all making up $62.6 \%$ of total) of research emanated from the Southwestern zone of the country. Majority, (44\%) of the Southwestern studies,focused on Infectious/Parasitic diseases; $29 \%$ were on reproductive health interventions; and $8 \%$ were on CV interventions. There were seven Southeastern CER studies which addressed Infectious/Parasitic diseases (28.6\%), CV diseases (28.6\%) and Reproductive Health conditions (14.3\%).

There were 13 studies from the South southern zone, 53.8\% of these addressed Infectious/Parasitic diseases, while $15.3 \%$ focused on Reproductive health.Sixty-seven percent of the studies from the North central zone were on Infectious/Parasitic conditions. Most studies from the Northeastern zone focused on the same area. 
Studies on management of Envenomation conditions(from snake bites) formed $67 \%$ of Northwestern articles (Figure 4). There was only one joint Southeastern/Southwestern study.

Trend in CER in Nigeria over 35 years:

No consistentCER trend was apparent for any of the broad groups of diseases and conditions from 1975 to 2010. Frequency of infectious disease studies waxed and waned with increasing up thrust from 1989 to 2009, followed by a sharp dip. Reproductive health CER studies show a decreasing turnover with time, howbeit with less flux.Frequency of CER studies for other categories was largely sporadic (Figure 5).

Types and Endpoints of Interventions:

Analysis of the intervention types showed that a preponderance $(62.6 \%, \mathrm{n}=62)$, of the interventions aimed at achieving cure of a condition; 29.3\% ( $\mathrm{n}=29)$, aimed toward prevention (Table 2). Most studies $(94.9 \%, \mathrm{n}=94)$ had interest in efficacy of treatment; approximately $60.6 \%(\mathrm{n}=60)$, had an adverse-effect component among the endpoints. Treatment failure was investigated in $28.3 \%(\mathrm{n}=28)$ of studies, while 12 studies (12.1\%) probed into patients' compliance with treatment regimen, only a few $(6.1 \%, \mathrm{n}=6)$ considered cost and acceptance $(4.0 \%, \mathrm{n}=4)$ of intervention by the patient/target population. No study investigated change in any indices of quality of lifesubsequent to intervention (Table 3 ).

Key outcome variables (or endpoints) were also explored according to broad categories of diseases/conditions and a few specific conditions. Most intervention studies (97.9\%) on infections and parasitic diseases focused on efficacy of intervention, $45.8 \%$ accommodated adverse-effects of intervention and $35.4 \%$ addressed treatment failure (Figure 6).

The reproductive health CER studies focused mainly on efficacy (95.8\%), adverse effects (75\%), and treatment failure (41.7\%). Only 25\% addressed compliance and $12.5 \%$ addressed acceptance. The cardiovascular studies addressed efficacy (100\%), adverse effects $(87.5 \%)$ and compliance $(12.5 \%)$ (Figure 6).No stable funding structure could be identified for these studies.

\section{Discussion}

The higher proportion of studies in the area of communicable diseases and infections is in keeping with the epidemiological profile of many developing countries where diseases consistent with economic deprivation place heavy burden on the population. Malaria has a high endemicity in this study area and expectedly studies on malaria constitute $23 \%$ of all articles reviewed in this paper. It is estimated that the number of fever/malaria episodes per person per year is 1.5 for children less than 18; months 3.5 for children under 5 years; and 0.5 for children 5 years and older yielding a total of $70-110$ million clinical cases per year ${ }^{(13-14)}$. The overall malaria mortality rate is 156 per $100,000^{(14)}$. The ubiquitous nature of the malaria scourge may well be an incentive for interest. Curiously studies on HIV/AIDS are much lower, $3 \%$ overall. With the ongoing PEPFAR programme aimed at scaling up access to free medication ${ }^{(15)}$ it may not be out of place to expect a commensurate increase in interest in HIV/AIDS studies in the future. Only one study explored tuberculosis. Evidence of some focus on reproductive health studies is also apparent. High indices of birth rate (41/100000), maternal mortality (1100/100000), with concurrent low contraceptive prevalence of $14.7 \%$ are well documented ${ }^{(16-18)}$. Cardiovascular disease studies, with hypertension taking the lead, are seen to be another area of interest for comparative intervention studies.

Official documents show that there exists a North/South dichotomy in socioeconomic and health indices ${ }^{(14,18)}$; and one may assume that health, treatment and research goals would vary according to the zonal setting ${ }^{(19)}$. Every geopolitical zone in Nigeria has several tertiary health facilities with skilled staffshaving relevant background toconduct intervention studies. The highest volume of included studies emanate from the Southwestern zone, and the least from the Northwestern zone. Only one study was jointly conducted in two zones. The CER pattern is consistent for most geopolitical zones, with infectious/parasitic disease studies showing dominance followed by reproductive health studies. The Northwestern zone however has a greater share of studies on Envenomation.

The trend in Nigerian CER studies from 1975 to 2010 has not revealed any consistent pattern. From years 1995 to 2009 there is seen a gradual buildup of CER studies on infectious/parasitic diseases only to be succeeded by a deep plunge after 2009. Most of these studies were malaria studies and they may reflect the drive to find reliable drugs for achieving clinical and laboratory cure following the emergence of widespread drug resistance by the malaria parasite. Going by the overall low volume of studies in this area it's hard to tell if the pattern seen for infectious/parasitic diseases reveals any true trend.

Most interventions were directed toward achieving cure,and there is a strong mismatch between the overall numbers of curative and preventive intervention. A peculiar find in health service delivery in Nigerian is the bloatedfocuson curative treatments to the detriment of prevention. Resource limitations should inform research into viable, effective and efficient methods ${ }^{(20)}$. However, this logic does not seem to reflect in the small number of studies that performedeconomic evaluations. Most studies address the issue of intervention efficacy, and a higher proportion of studies addressed adverse effects/tolerability. Treatment failure was considered in some studies, and a few considered user/patient compliance with therapy. This review showed that although 
Comparative Effectiveness Research (CER) In Nigeria: The Milestones And The Millstones.

most infectious disease studies addressed efficacy, few considered adverse-effects and treatment failure as endpoints, whilemore malaria studies (60.9\%) addressed treatment failure which is in keeping with concerns about drug resistance. A similar scenario is emerges for reproductive health studies. Cardiovascular studies emphasized efficacy and adverse-effects.

\section{Conclusion}

In conclusion, the overall volume of intervention studies was low. A greater of the proportion of studies, $48.5 \%$, investigated treatment options for infectious and parasitic diseases; $24.2 \%$ investigated reproductive health interventions; $62.6 \%$ of interventions aimed to achieve cure, and $29.3 \%$ aimed for prevention. Study endpoints were efficacy $(95.9 \%)$, adverse effects/tolerability (60.6\%), treatment failure (28.6\%) and cost (6.1\%). Patterns were similar in different zones of the country. CER does reflect the pattern of need and the dominant type of healthcare in Nigeria.

In preparing this article, no funding support was received from any source.

\section{References}

[1]. Golub RM, Fontanarosa PB. Comparative Effectiveness Research Relative Successes. JAMA. 2012;307(15):1543-645.

[2]. Docteur E, Berenson R. How Will Comparative Effectiveness Research Affect Quality of Health Care? Timely Analysis of Immediate Health Policy Issues 2010.

[3]. Herrick LM, Locke GR, Zinsmeister AR, Talley NJ. Challenges and Lessons Learned in Conducting Comparative-Effectiveness Trials. The American Journal of Gastroenterology 2012;107:644-9.

[4]. Daniel T, Leonard MA. Comparative Effectiveness and Real-World Evidence. Am J Managed Care 2010;16( 6):410-1.

[5]. Alemayehu D, Ali R, Alvir JMJ, Cappelleri JC, Cziraky MJ, Jones B, et al. Examination of Data, Analytical Issues and Proposed Methods for Conducting Comparative Effectiveness Research Using “Real-World Data”. JMCP. 2011 Supp1 17(9a):S2-S37.

[6]. US CBO. Research on the Comparative Effectiveness of Medical Treatments: Issues and Options for an Expanded Federal Role. Washington D C. U. S. A. 2007 p. 1-35

[7]. IOM. Initial National Priorities for Comparative Effectiveness Research. Institute of Medicine (IOM): National Academy Press, Washington DC; 2009.

[8]. Willke RJ, Mullins CD. "Ten Commandments" for Conducting Comparative Effectiveness Research Using "Real-World Data. JMCP 2001;Suppl 17(9-a ):S10-S3.

[9]. Oxman AD, Lavis JN, Fretheim A, Lewin S. Support Tools for Evidence-Informed Health Policy Making (STP) 17: Dealing with Insufficient Research Evidence. Health Research Policy and Systems 2009;7(Suppl 1):S17.

[10]. Manchikanti L, Falco FE, Boswell MV, Hirsch JA. Facts, Fallacies, and Politics of Comparative Effectiveness Research: Part I. Basic Considerations. Pain Physician 2010;13:E23-E54.

[11]. Sox H, Helfand M, Grimshaw J, Dickerson K, Tovey D, et al. Comparative Effectiveness Research: Challenges for Medical Journals. PLoS Med 2010;7(4):e1000269.

[12]. FMOH. Revised National Health Policy. Abuja, Nigeria2005.

[13]. FMOH. Strategic Health Plan 2009-2013: National Malaria Control Programme. Abuja, Nigeria 2008.

[14]. Roll Back Malaria: Country Needs Assessment. Malaria Consortiun (MC) 2008.

[15]. PEPFAR. The President's Emergency Plan for AIDS Relief General Policy Guidance for All Bilateral Programs. Office of the U.S. Global AIDS Coordinator. 2005.

[16]. PCB. 2009 World Population Datasheet. Population Reference Bureau (PCB) 2009.

[17]. WHO. World Health Statistics 2010. Geneva, Switzerland: World Health Organization (WHO).

[18]. NPC. Nigeria Demographic and Health Survey 2008. National Population Commission of the Federal Republic of Nigeria. Abuja, Nigeria 2009.

[19]. Manchikanti L FF, Boswell MV, Hirsch JA. Facts, Fallacies, and Politics of Comparative Effectiveness Research: Part I. Basic Considerations. Pain Physician 2010;13:E23-E54.

[20]. Chandra A JA, Skinner JS. The Pragmatist's Guide to Comparative Effectiveness Research. J Econ Perspect 2011;25(2):27-46.

Table 1. Frequency of Studies by Broad Category

\begin{tabular}{ll}
\hline Broad Group & Frequency $(\%)$ \\
\hline Allergy/Atopy & $3(3.0)$ \\
Cardiovascular Diseases & $8(8.1)$ \\
Degenerative & $3(3.0)$ \\
Dental Health & $3(3.0)$ \\
Envenomation & $3(3.0)$ \\
Food and Nutrition & $2(2.0)$ \\
Infectious Diseases/Parasitic Infestations & $48(48.5)$ \\
Malignancies & $1(1.0)$ \\
Mental Health & $1(1.0)$ \\
Opthalmic conditions & $2(2.0)$ \\
Reproductive Health & $24(24.2)$ \\
Trauma/Pain & $1(1.0)$ \\
Total & $99(100)$ \\
\hline
\end{tabular}




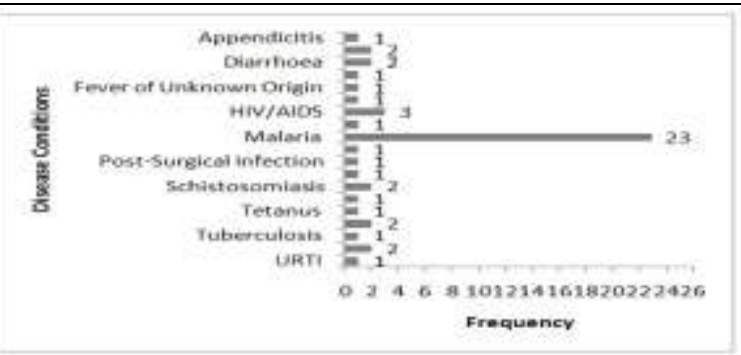

Figare 1. Propertion or Iafectious Disease/Parasitic Infestations studied

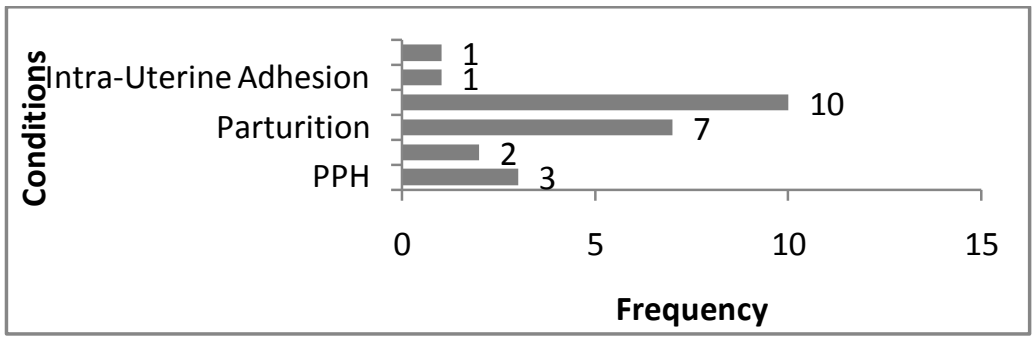

Figure 2. Aspects of Female Reproductive Health Conditions Studied

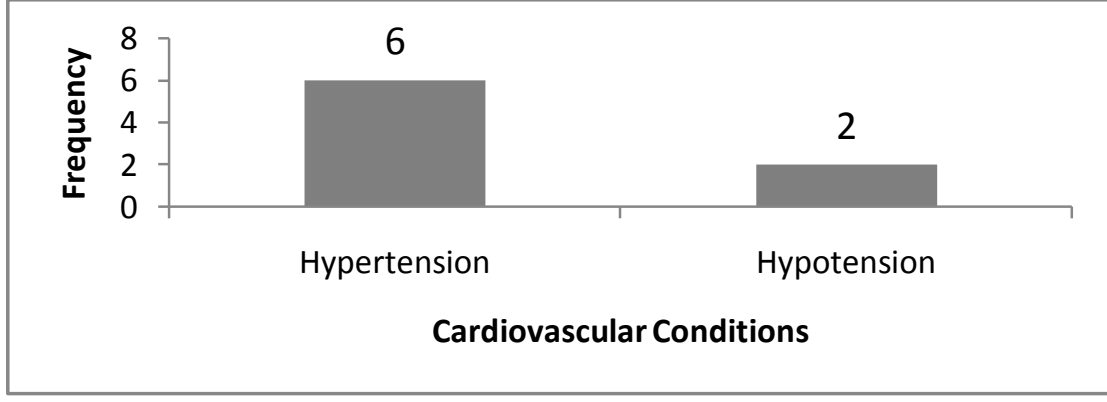

Figure 3. Aspects of Cardiovascular Conditions Studied

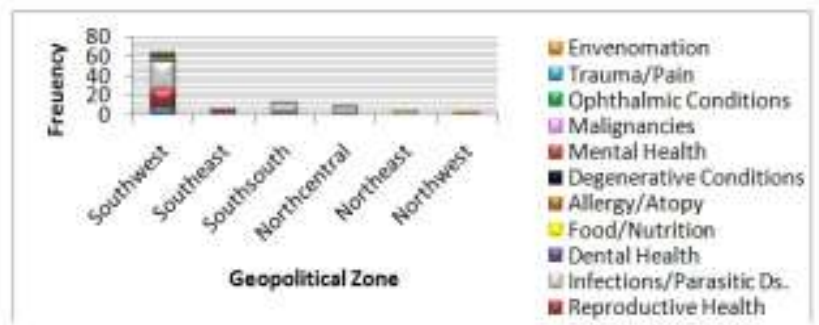

Figure 4. Disease Conditions Investigated According to Geopolitical Zone

Each stacked column shows specific disease conditions on which CER studies were conducted in a particular geopolitical zone. The length of the segment in each column corresponds with the absolute frequency of CER studies on the disease condition so represented.

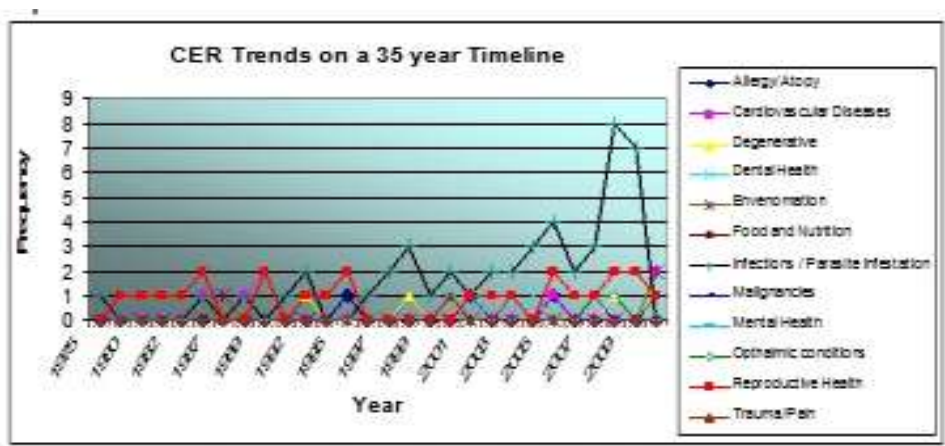

Figure 5. A 35 year Trend of CER Studies in Nigeria 
Comparative Effectiveness Research (CER) In Nigeria: The Milestones And The Millstones.

Table 2. Types of Interventions

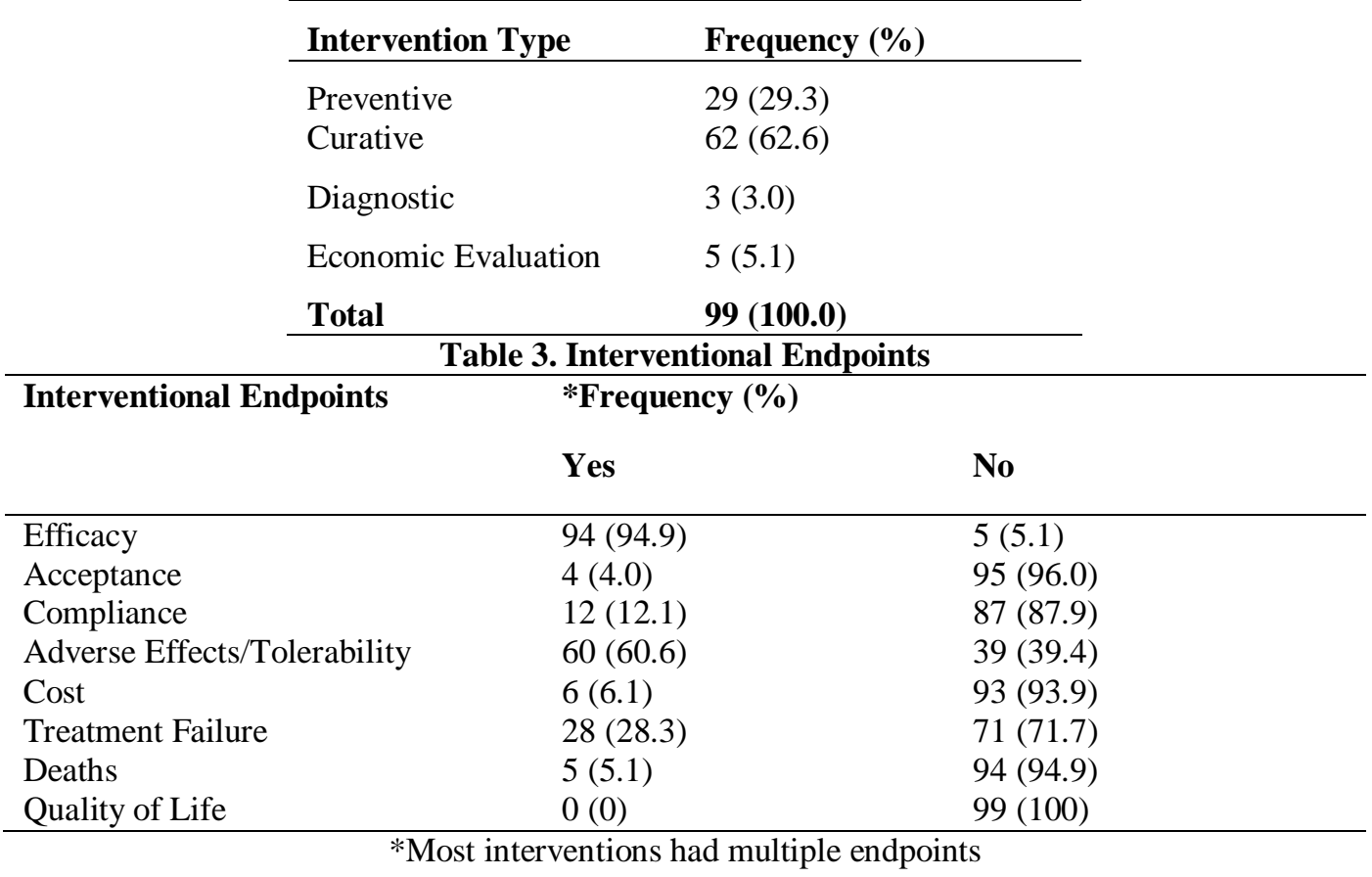
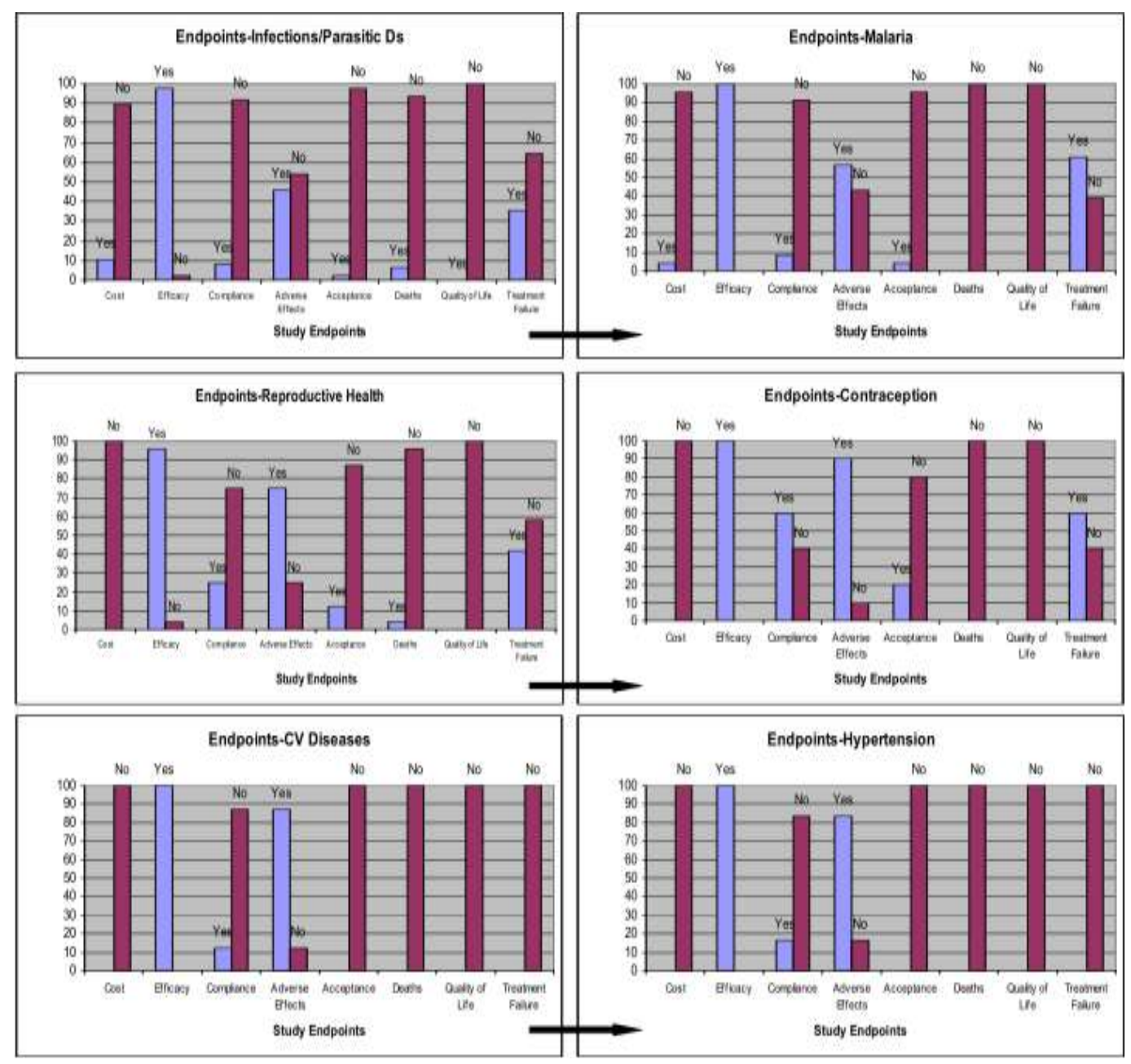

Figure 6. Intervention Endpoint According to Broad and Specific Disease Categories

Diagram shows study endpoints for a major disease category and the corresponding most frequently researched disease condition within that category (as indicated by the arrows) 